\title{
Ontological Description and MAS based Composition Process of SWS's for Recruitment Domain
}

\author{
Alankar Srivastava \\ Computer Science \& Engg. \\ Department \\ SITM, Barabanki, India
}

\author{
Shruti Saxena \\ Computer Science \& Engg. \\ Department \\ SRMCEM,Luknow,India
}

\author{
Chandrabhan Singh \\ Computer Science \& Engg. \\ Department \\ SITM, Barabanki, India
}

\begin{abstract}
It seems that information is spread over internet and to retrieve useful information easily by mean of intelligent computing is the growing field of research. This need gives birth to semantic web services and various approaches are given for composition of these services. The key problem is to fulfill user's request. Intelligent Agents are used as a powerful approach for semantic web services selection and composition. Agent can be viewed as a self contained autonomous software component or say codes which is used for fulfillment of users request in any domain. Here in this paper semantic web services and agent capability is used for Man-Power Recruitment System (MPRS). Ontology of domain is used as a complete domain knowledge. Tools like Protégé is used to create agent profile which shows the ontological description of recruitment domain. This paper focuses on how multi-agent system provides a new variety for recruitment system. This paper also spotlights to show an interactive recruiter for Man-Power Recruitment System (MPRS). Here service selection, task decomposition and service composition process of semantic web is exposed on the basis cognitive parameters, predilection and situation for man-power recruitment system.
\end{abstract}

\section{General Terms}

Semantic web services, SWS Composition, Agent, Ontology and Recruiter.

\section{Keywords}

Man-Power Recruitment System, Recruiter, Man-Power Recruitment (MPR) Domain Ontology, Agent, Semantic Web.

\section{INTRODUCTION}

Semantic web which becomes the extension of current web is not only web of linked pages but it show intelligence also and viewed as a huge collection of data. The emergent use of XML over network prominence on the exploit of XML based indexing as querying language to build web semantic. In recruitment system this semantic attribute can also be used for not only retrieving information over internet but also it can be utilized for training and generating a feasible process of recruitment better for both company and individual. In recruitment process of any company the problem that comes in picture are like where the cost of selection will be minimum i.e. number of eligible candidate will be high, secondly from where we will get candidates which will be suitable in the working environment of the company and various other parameters from the point of view of company. Here in manpower recruitment system the aim is to provide complete compiled information to company or say selectors about college, zone, students, their back log, average percentage, collage reputation and other factors about collage at a single point. It is well-known that semantic web provides a podium for data amalgamation, distribution and reusing from various sources or services. Here the complete domain knowledge is represented in form of ontology's. Control of semantic web [1] with potentiality and capacity of agent machinery $[2,3]$ is used to assist man-power recruitment domain. Distributed problem solving approach [4] of multi-agent system is used for semantic web services and then the compositions of these services are done to fulfill the task. A variety of researches are made on SWS and its composition [5, 6]. Data in current web is stored in variety of languages over internet but semantic web provide a unique XML based web description language known as RDF [7]. It has been seen that RDF and OWL [8] are mostly used for depiction of resources and how they are connected on web. This paper focuses on joint approach of multi-agent and SWS's to assist recruitment system. The combined circumstances of multi-agent and SWS's can be capitalized in various domains like medical, education planning, intelligent tutoring etc. This paper paying attention on recruitment system.

\section{COGNITION BASED AGENT SELECTION}

Selection of agents is an important task which involves selection of both coordinator as well as service facilitator agents. This selection process is done on the basis of cognitive parameters. These parameters are user's preferences, quality factors and pre-conditions. In man-power recruitment domain [7] these parameters are average student percentage in the college, previous placement ratio, average backlog per student, state ranking of college, level of aggradations of college, faculty grading etc. Firstly the input request of company is fetched then verification and validation for that input request is done. Then on the basis of various cognitive parameters the QoS of agent is evaluated. Agent having best QoS value is selected as coordinate agent. Selection of facilitator agents is based on decomposition process. When large or say complex input request which is not possible to fulfilled by using single agent then that request is decomposed in various less dependent sub-tasks and for each sub-task a service provider or say facilitator agent is selected. Selection of semantic value can be formulize as QoS of agent.

$\mathrm{Q} \propto S$ of Agent $\propto \mathrm{Pf}^{*} \mathrm{Qh}{ }^{*} \mathrm{C} / \mathrm{D}$

where $\mathrm{Pf}=$ placement factor of college

$\mathrm{Qh}=$ quality of service for hospital

$\mathrm{C}=$ charge of service

$\mathrm{D}=$ zonal distance 


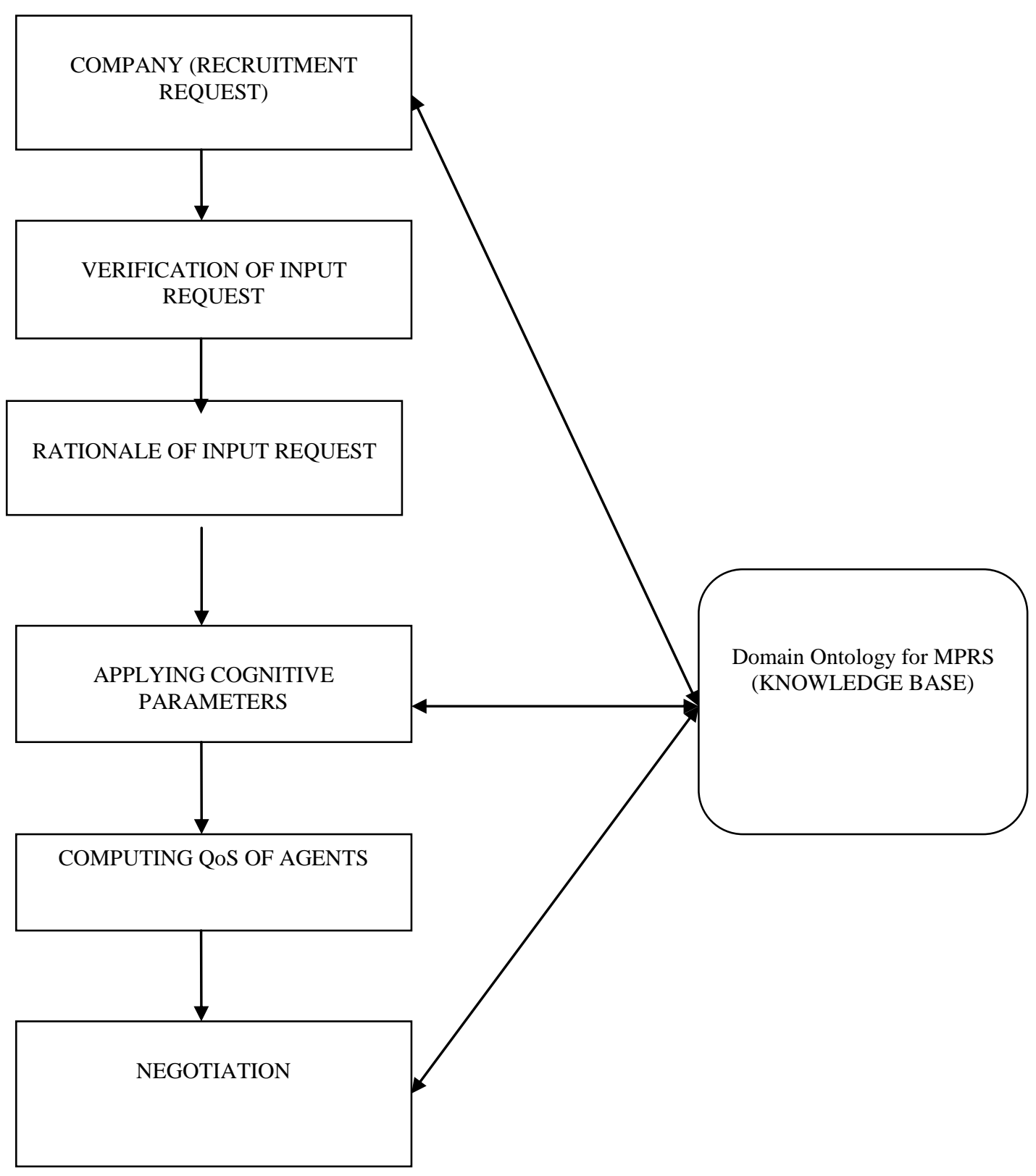

Fig 1: Selection Process of Agents

\section{ONTOLOGICAL DISCRIPTION OF MPRS}

It is known that ontology is a pictorial representation of complete domain knowledge. Web Tools like Protégé [10], Jena etc are used for the development of agents profile in languages like RDF/RDF-S [8] or OWL [9]. For Man-power Recruitment system Protégé [10] is used to create complete domain ontology. The ontological graph for that is shown in figure 4. That graph demonstrates relationship between subject and object. The ontological graph contains complete information /knowledge about that domain. Has College, has Branch, has Student, has Backlog, is College of, is Student of, etc are various object properties which are created between objects and subjects. Figure 2 shows the class hierarchy and figure 3 shows the object properties discussed above. Various classes like zone, city, college, branch, TPO etc are used to create class hierarchy. 


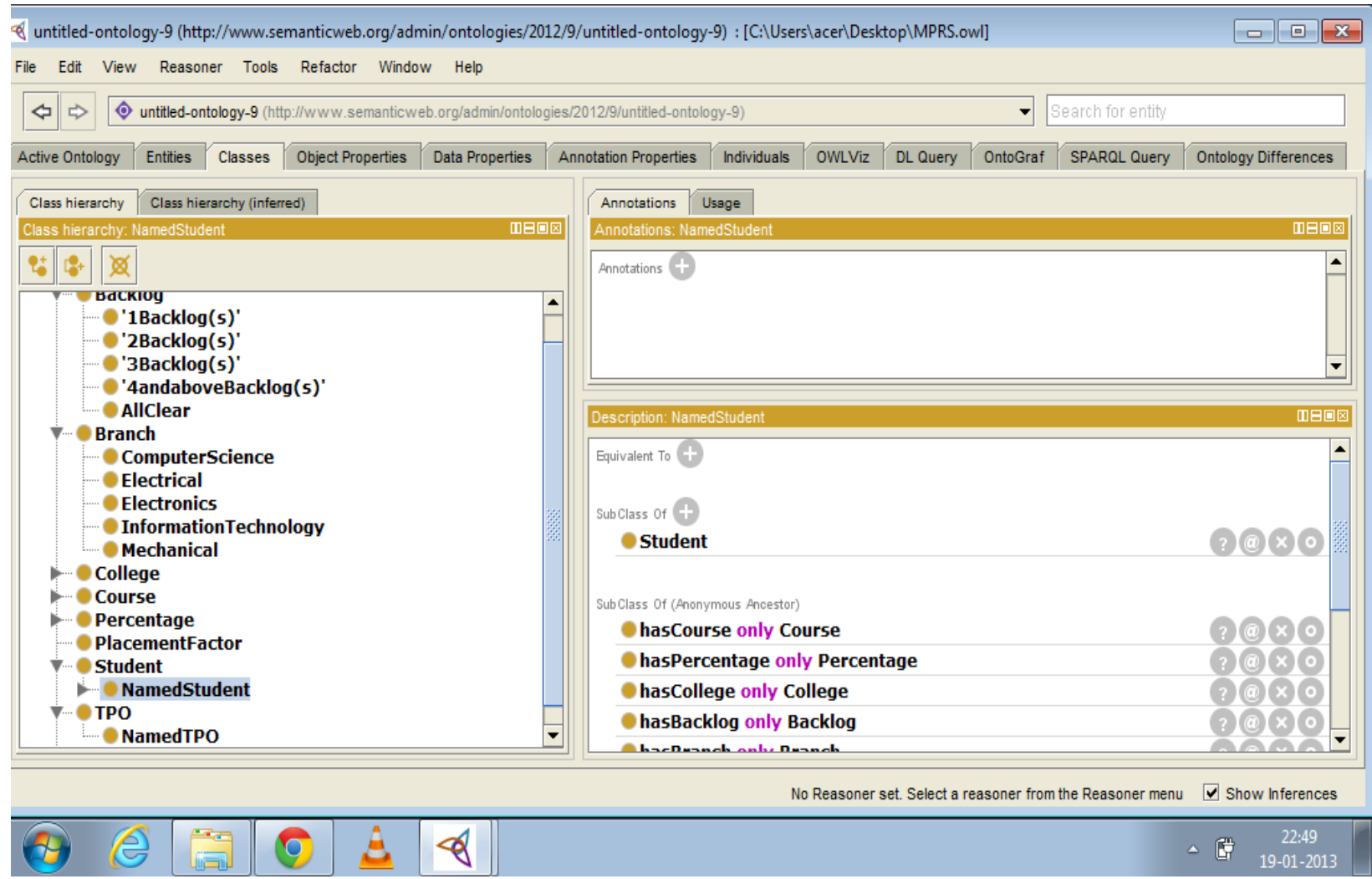

Fig 2: Class Hierarchy of MPRS

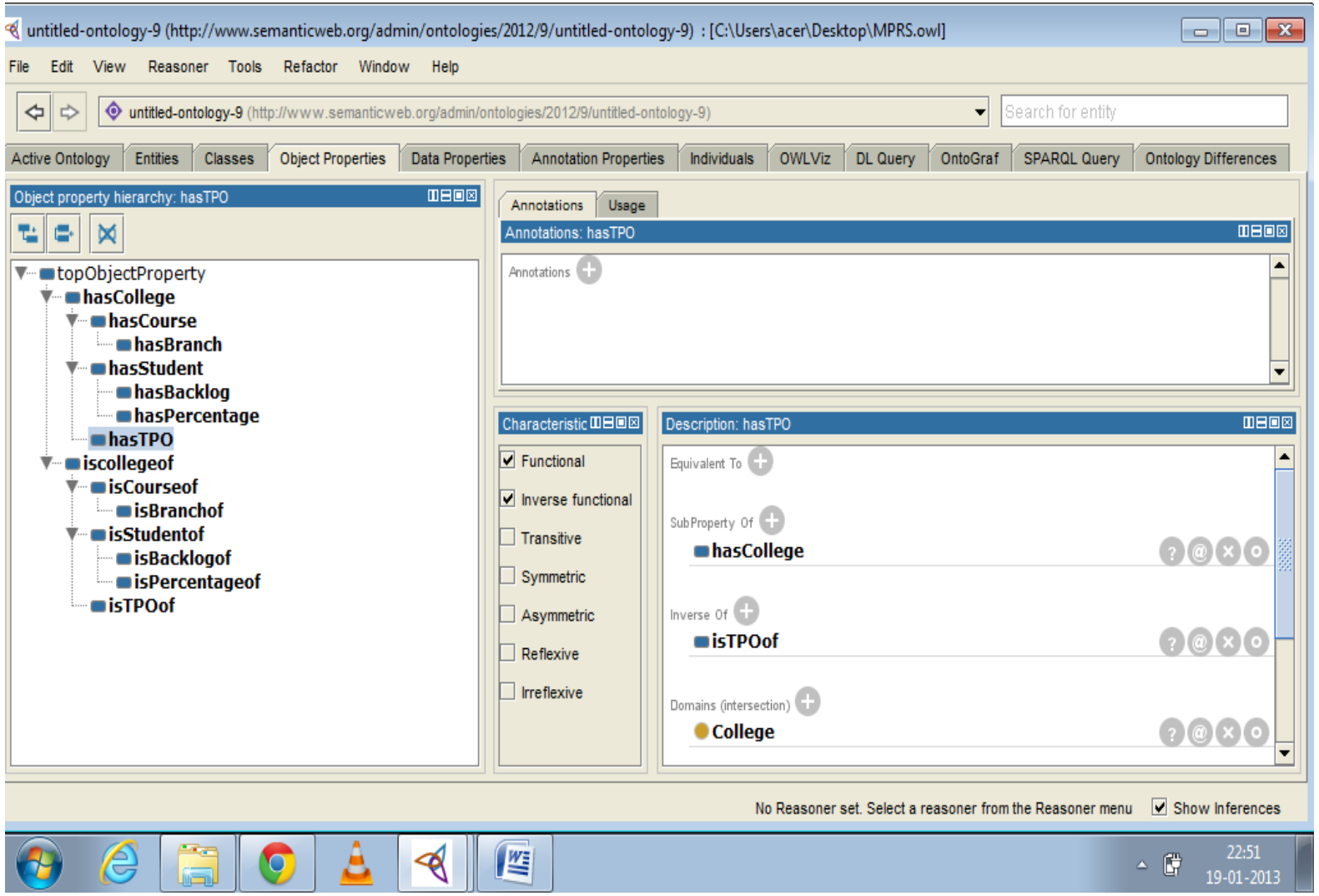

Fig 3: Object Property of MPRS 


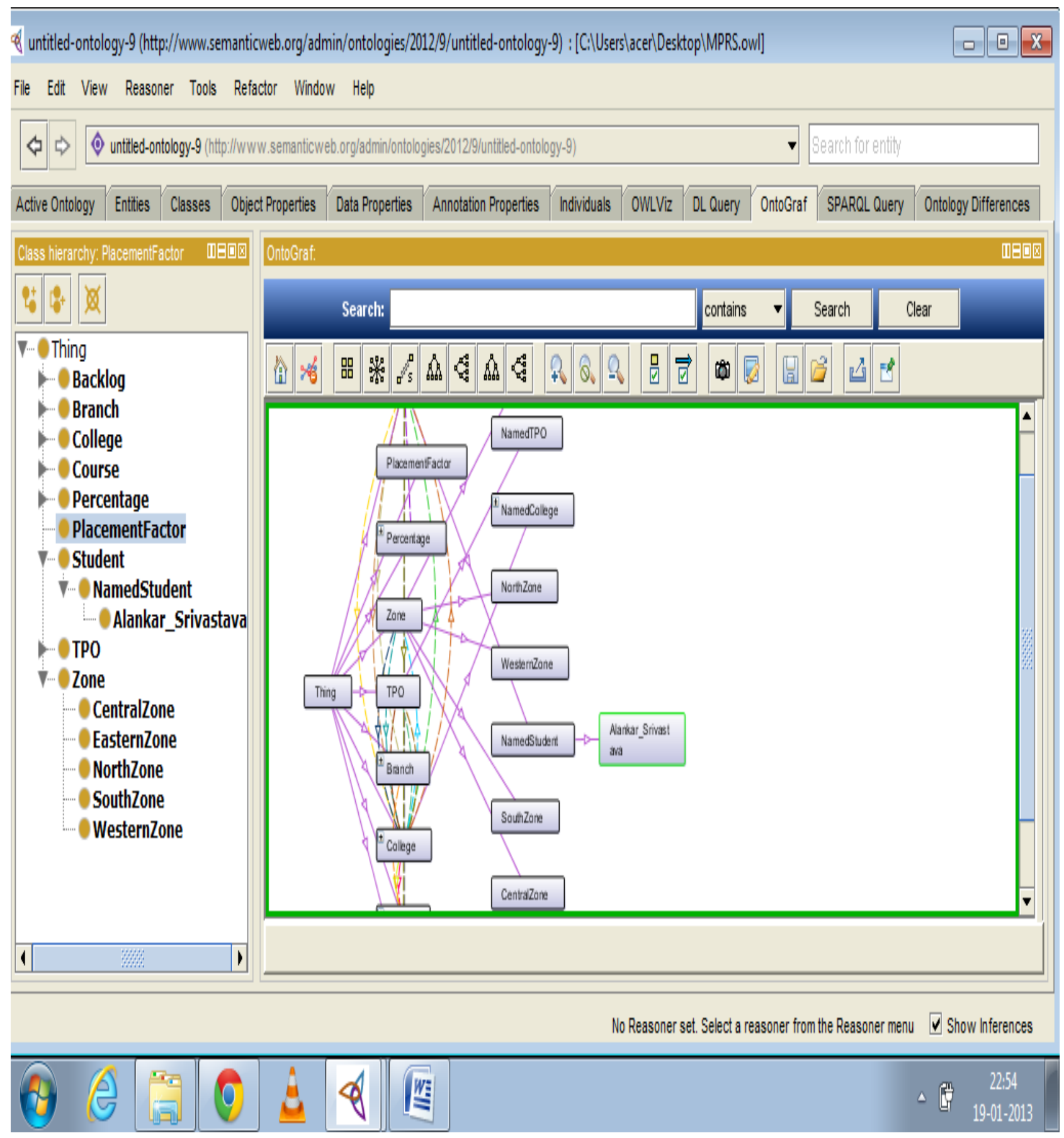

Fig 4: Onto-Graph of MPRS

\section{DISCOVERY AND SELECTION PROCESS OF SWS'S FOR MPRS}

To decompose as complex task in several sub tasks is an important issue. These sub tasks are partially or say fully independent. These sub-task can be treated as fully or say partially independent tasks. If there is any dependency agents communicate to each other with their sociable propriety. For each sub task a service facilitator agent is provided and on the basis of pre-conditions and preferences for each task service is selected. Users specified these pre-conditions and preferences and when a service is selected on the basis on these preconditions and preferences the user approve solution.
The decomposition of tasks for recruitment system is shown in figure 5. This will show how company request is proceed by service caller agent and then service facilitator agent work on it in order to find appropriate solution of that complex problem. The company input request is decomposed in various sub tasks like recognize zone, recognize state, recognize college, contact TPO, arrange schedule etc. Decomposition and selection process is shown in figure 5 . On getting solution of all input sub-tasks company use to approve the solution then composition process take place to find aggregate of all these web services in order to produce final solution. Thus composition process becomes an important aspect of user's complex problem and to get easily a complete solution. 


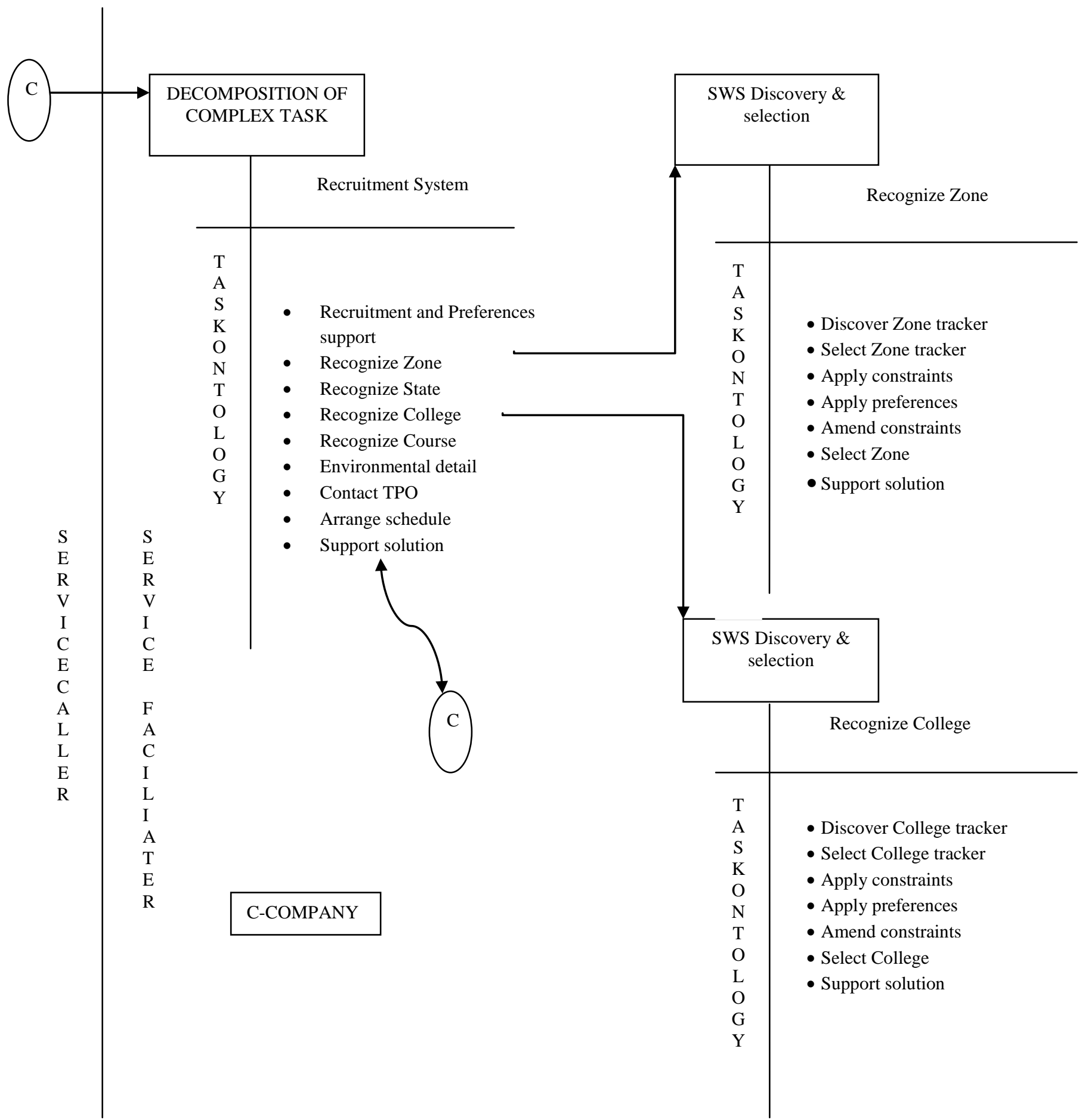

Fig 4: Semantic Web Service composition for MPRS

\section{CONCLUSTION}

The whole Paper is based on semantic web service composition for man-power recruitment domain for providing used more dynamic data and using the web as retriever rather than locater of resources. Ontologies are used as domain knowledge. Protégé is seems to be an important semantic web development tool and tool which can be easily used to create profile. Distributed problem solving approach of multi agent system is used to decompose complex task in sub tasks. This paper presents a model which shows how agent's power can be used to provide a aggregate web service which gives full information to company or say selectors about college, zone, students, their back log, average percentage, college reputation and other factors about college at a single point. This will also reduce the cost of recruitment and will provide more suitable candidates which 
reduces the attrition rate of the company. In future this system focuses on other fields rather than engineering and facilitates recruitment process easier and better.

\section{REFERENCES}

[1] Berners-Lee, T., Hendler, J., and Lassila, O., 2001 the Semantic Web. Scientific American, 284:34-43.

[2] Ermolayev V., N. Keberle, Sergey P., Kononenko, Terziyan towards a Framework for Agent-Enabled Semantic Web Service Composition, Int. Jl. Of Web Services Research, 1(3),pp 63-87.

[3] Wooldridge, M. An introduction to Multi agent system. Wiley Ed. (2002).

[4] Durfee, D.H. Distributed problem solving and planning. In [1] 121-164.

[5] Sandeep Kumar, and R. B. Mishra, Semantic web service composition IETE Technical Review vol 25. No.3 May-June, (2008) pp 105-121.
[6] Chandrabhan Singh, Sanjay Sachan and Mohit Gangwar. Article: MAS based Selection and Composition Process of SWS's for Medical Health Care Planning System. International Journal of Computer Applications 60(16):40 44, December 2012. Published by Foundation of Computer Science, New York, USA.

[7] Alankar Srivastava, Chandrabhan Singh, Mohit Gangwar "Utilization of Multi Agent System for MPRS" presented in International Conference on Artificial Intelligence and Soft Computing (AISC 2012) at IIT-BHU on 7th - 9th Dec 2012.

[8] Hernam, I., Swick, R. and Brickley, D., Resource Description Framework (RDF), W3C Website, http://www.w3.org/RDF.

[9] McGuiness, D.L, Harmelen, F.V. OWL Web Ontology Language Overview, http://www.w3.org/TR/owl-features.

[10] Stanford Center for Biomedical Informatics Research. Protégé, http.stanford.edu/.

I. $\mathrm{s}$ 